\title{
El Rol de los Estilos de Liderazgo en la Implantación de la Responsabilidad Social Como Filosofía del Nuevo Modelo Post-Crisis
}

\author{
Cecilia de la Corte \\ Ana Carrasco González*
}

ResUMen:

En la actualidad, la percepción de las empresas como meras creadoras de beneficio económico empieza ya a estar superada. Una nueva concepción de las organizaciones se está extendiendo y la Responsabilidad Social es quizás la más evidente de sus manifestaciones. En este panorama el estilo de liderazgo que ejercen los directivos de las organizaciones juegan un papel fundamental, puesto que ellos son quienes marcan las estrategias y las lineas a seguir para alcanzar sus objetivos. El presente artículo pretende hacer una reflexión acerca de la situación económica-social que azota a España y plantea la necesidad de asentar el nuevo modelo post-crisis sobre criterios éticos de Responsabilidad Social, cuyo desarrollo e implantación requiere de estilos de liderazgos comprometidos con la excelencia empresarial, la globalización y el cambio.

Palabras Clave:

Responsabilidad social, liderazgo, crisis económica, organizaciones saludables.

ABSTRACT:

Currently, the perception of companies as merely creating economic benefit is already beginning to be overcome. A new concept of organization is expanding and Social Responsibility is perhaps the most obvious manifestations. In this scenario

* Departamento de Psicología Clínica, Experimental y Social. Universidad de Huelva • anacar@uhu.es 
the style of leadership exercised by managers of organizations play a fundamental role, since they are the ones who make the strategies and guidelines to follow to achieve their goals. This article aims to reflect on the social-economic situation plaguing Spain and a need to establish the new post-crisis model of ethical social responsibility, the development and implementation requires leadership styles committed to excellence business, globalization and change.

KEYWORDS:

Social responsibility, leadership, economic crisis, health organizations.

\section{INTRODUCCIÓN}

En la actualidad, la percepción de las empresas como meras creadoras de beneficio económico empieza ya a estar superada, especialmente ante la situación de crisis en la que aún se encuentra España y que está provocando consecuencias a todos los niveles. El año 2011 ha estado condicionado por la situación económica mundial, la cual reduce los márgenes de reacción de todo lo que se afronta, especialmente porque la crisis no sólo es financiera sino que cada vez se está haciendo más profunda a nivel social, provocando incluso importantes repercusiones en la salud de las personas.

En este contexto general, una nueva concepción de las organizaciones se está extendiendo y la Responsabilidad Social (RS) es quizás la más evidente de sus manifestaciones por los efectos que conlleva sobre la productividad y la competitividad de las empresas y sus equipos de trabajo, así como en la creación y mantenimiento del empleo, principal reto en la Agenda Europea 2020.

Tal como expone González (2003), en todo este panorama los directivos de las organizaciones juegan un papel fundamental, puesto que ellos son quienes marcan las estrategias y las líneas a seguir para alcanzar sus objetivos. Por tanto, se presupone que el estilo de liderazgo que ejerzan condicionará en gran medida el desarrollo de la empresa, respecto tanto a su productividad como en el ejercicio responsable socialmente en el contexto en el que interactúen.

Así pues, el objetivo del presente artículo consiste en ofrecer una visión general de la situación económica-social de España y de la importancia de la RS como pilar base de desarrollo para superar la actual crisis, analizando el rol que deben ejercen los directivos de las organizaciones y los estilos de liderazgo más idóneos para la implantación de estrategias socialmente responsables.

\section{CONTEXTO GENERAL DE LA CRISIS ESPAÑOLA}

García-Trevijano (2011) señala que como en los fenómenos naturales, los efectos económicos de la perturbación inicial crean otros efectos expansivos de la crisis, 
que no siendo constitutivos de la misma, aumentan progresivamente el movimiento destructivo. De esta forma, las tres causas que han generado la crisis actual: la inmobiliaria, la financiera y la bursátil, que tienen de común su carácter especulativo y mutuamente atractivo, han provocado efectos en el terreno institucional de lo social y lo político (sindicatos, partidos y gobierno), que es donde se manifiesta con mayor virulencia, por la reacción de los particulares sectores afectados por la crisis y de la sociedad civil en general.

Respecto a los efectos políticos, la consecuencia directa ha sido la caída de la actividad económica, teniendo como consecuencia el incremento del paro. El Instituto Nacional de Estadística nos ofrece la tasa de paro en el tercer trimestre del año 2011, que ha crecido con respecto al segundo trimestre, pasando de 4.833 .700 personas desempleadas a 4.978.300 parados, es decir, el 21,52\% (Encuesta de Población Activa). Estas cifran se ratifican con el aumento de la tasa de mortalidad de las PYMES españolas que constituyen el 99,88\% de las que conforman el censo, excluida la agricultura y la pesca, habiéndose reducido en 2009 el 2,7\% de éstas por la crisis.

El desempleo no sólo supone la interrupción de los ingresos, sino que también implica la pérdida de la identidad individual y social, el cambio de estatus, la reducción del bienestar, la descalificación y con frecuencia una pérdida de confianza en sí mismo, tal y como señalan Alabort, Buendia, Fuster, Obispo, y Rodríguez (2008). Buena prueba de todo ello es que al aumentar el desempleo se incrementan también el número de suicidios, los malos tratos infantiles, las desavenencias conyugales, los problemas familiares y las enfermedades mentales o el número de infartos y ataques de corazón. Asimismo, aumentan el número de personas que cometen delitos, como robos callejeros u otros para cubrir necesidades básicas, acrecentando de esta forma las personas que pierden la libertad ingresando en centros penitenciarios.

Es decir, los efectos psicosociales de la crisis económica son evidentes, pues generan un creciente malestar subjetivo en la medida en que se vive como una amenaza al nivel de vida y consumo o a las mismas posibilidades de sobrevivencia, generando frustración, tensión y descontento. Asimismo, el acrecentamiento de los contratos temporales y la transformación de la economía (automatización, restructuración, privatización, etc.) están incrementando el fenómeno social del "Job Insecurity" o trabajo inseguro, concepto expuesto por Hans de Witte (2011) y que hace referencia a la percepción subjetiva que tiene el trabajador de la pérdida involuntaria del empleo, lo que conlleva graves consecuencias para los individuos, ya que afecta negativamente a la satisfacción y al bienestar con el paso del tiempo, convirtiéndose en una fuente de estrés crónico (Garst, Frese, \& Molenaar, 2000) y en consecuencia, para las empresas e incluso para la sociedad.

El trabajo es la llave para la integración y la participación social porque ofrece ingresos, contactos sociales, desarrollo individual y estatus y reconocimiento social, 
entre otros. Por tanto, sus consecuencias especialmente ahora con la crisis económica, donde la inseguridad en los puestos de trabajo alcanza altos índices, pues la tasa de ansiedad por inseguridad en el empleo en España ha pasado del 14\% en 2005 al 16\% en 2010 (V Encuesta Europea de las Condiciones de Trabajo), son nefastas para aquellos que siguen trabajando pero sin la certeza de mantener sus empleos en el tiempo, generando una percepción impredecible e incontrolable por la violación del contrato psicológico entre trabajador y empresario.

Esta situación en el seno empresarial está provocando a su vez un aumento de los riesgos Psicosociales que perjudican a la salud de los trabajadores y trabajadoras, causando estrés y a largo plazo enfermedades cardiovasculares, respiratorias, inmunitarias, gastrointestinales, dermatológicas, endocrinológicas, musculoesqueléticas y mentales, consecuencias de unas malas condiciones laborales, concretamente de una deficiente organización del trabajo y no tanto de las circunstancias personales y familiares, perjudicando al capital humano y psicológico de las plantillas (Alabort y cols, 2008).

A partir de todo lo expuesto, se infiere que la intervención en la crisis inicialmente económica que está teniendo consecuencias devastadoras en la esfera de lo psicosocial debido fundamentalmente a la reducción de la calidad de vida laboral y por ende, personal, requiere de una actuación inmediata. Como consecuencia, España está obligada a concentrar todos sus esfuerzos para salir de esta situación, no sólo por la propia necesidad que tiene el país de cambiar y progresar, sino por las directrices marcadas por la Unión Europea, quien en las recomendaciones del Consejo de julio 2010, cita textualmente: "Los Estados miembros deben realizar todos los esfuerzos posibles para cumplir sus objetivos nacionales y eliminar los obstáculos al crecimiento. Como parte de las estrategias generales de superación de la crisis económica, los Estados miembros deben aplicar programas ambiciosos de reforma para garantizar la estabilidad macroeconómica y la viabilidad de las finanzas públicas, mejorar la competitividad y reducir los desequilibrios macroeconómicos y mejorar los resultados de su mercado laboral" (p. 191/28).

La determinación del Gobierno a aplicar la reforma se confirma en el programa nacional de reforma y en el programa de estabilidad presentados a la Comisión el 29 de abril de 2011, documentos que también reflejan los compromisos a corto plazo que presentó España el 25 de marzo de 2011 en el marco del Pacto por el Euro Plus, con los objetivos de mejorar la competitividad de la economía española y contribuir a una mayor convergencia en la zona del euro.

González (2003) señalaba que el panorama del momento para la empresa no era nada alentador al igual que hoy en día, tal y como se ha analizado anteriormente con cifras, ya que nada favorece sus actividades, se atenta contra la estabilidad financiera, 
afecta el bienestar interno y externo y por ende, a la productividad repercutiendo en la competitividad. Asimismo, la productividad de las personas se ve seriamente afectada cuando se encuentra mermada emocionalmente y en consecuencia los resultados de la empresa. Por tanto, si el ambiente en que se encuentra la sociedad y la empresa es sano y favorable, la productividad y la competitividad serán mayores, pero de no ser así, se manifestará en sus resultados de producción, de mercadeo y financieros.

Esto produce decaimiento emocional y se convierte en razón para el ausentismo, la deserción laboral, la accidentalidad en el ejercicio de las funciones de trabajo, la apatía, el crecimiento de conflictos, la baja motivación por lo que se hace y demás manifestaciones que disminuyen la capacidad de concentración y la disposición hacia la productividad, además perjudica a aspectos como la perdida total de interés por lo que se hace y por la misma vida.

Las empresas son partícipes de la responsabilidad en algunos de los efectos mencionados o en todos, ya sea por sus políticas o por la carencia de las mismas, por sus sistemas de dirección y por una serie de costumbres y vicios empresariales que le alejan de una actuación responsable que mida las consecuencias de sus actos. La responsabilidad o la no responsabilidad, es parte de la cultura de una empresa. Pero, ¿qué se entiende por RS y cual es su importancia en este contexto?

\section{RESPONSABILIDAD SOCIAL: FILOSOFÍA DEL NUEVO MODELO POST-CRISIS}

Tal como expone Vargas Niello (2006), la delimitación de la responsabilidad de las empresas no es muy nítida, constatándose en la mayor parte de las instituciones internacionales que impulsan este tipo de iniciativas así como en la comunidad empresarial, cierto grado de imprecisión a la hora de expresar cuál es el significado de los conceptos asociados a este fenómeno.

A pesar de la profusión terminológica, se ha producido una paulatina integración de un conjunto de prácticas similares estructuradas en torno a la RS en una serie de ámbitos concretos: la responsabilidad medioambiental y social, la transparencia, el diálogo con las partes interesadas y el buen gobierno. Este esfuerzo ha posibilitado definiciones y propuestas que comienzan a ser coincidentes en sus estructuras básicas, de forma que las empresas están empezando a contar con referentes que orientan sus propios programas mediante estructuras consensuadas. De Castro Sanz (2005), comenta que cualquier definición de RS debe contar con al menos las siguientes variables:

- Ha de partir de forma explícita del cumplimiento de la legislación vigente, así como las normas internacionales aceptadas (OCDE, OIT, Derechos Humanos, ONU, etc.). 
- Ha de comportar compromisos éticos objetivos: surgen de la relación con sus grupos de interés (stakeholders) y con el entorno y del desarrollo de su actividad, también con la relacionada con otras empresas.

- Ha de explicitarse en la generación de impactos medibles o cuantificables, premiables o punibles, según la legislación, normativa vigente o políticas públicas de fomento de la que se es responsable.

Según lo comentado, el Libro Verde de las Comisiones Europeas la conceptualiza como: "La integración voluntaria, por parte de las empresas, de las preocupaciones sociales y medioambientales en sus operaciones comerciales y sus relaciones con el entorno. Ser socialmente responsable no significa solamente cumplir a plenitud las obligaciones jurídicas, sino también ir más allá de su cumplimiento, invirtiendo más en el capital humano, el entorno y las relaciones con los interlocutores" (Vargas, 2006: 17).

Haciendo un análisis más exhaustivo del impacto de la RS en las organizaciones, cabe destacar el doble impacto que De la Corte (2011) considera que tiene la RS sobre la reputación corporativa y sobre la reputación interna de la empresa, las cuáles deben constituir los dos pilares de actuación en el nuevo Modelo post-crisis.

Respecto a la primera de ellas, la reputación corporativa resulta de importancia para las organizaciones porque es uno de los elementos en los que se basa su capacidad competitiva, puesto que posibilita una mejor valoración de las personas y/o entidades ajenas a la empresa (Stakeholders externos), a través de un proceso de identificación de valores como el que se da en el contrato emocional. Además, permite la mejora de su posicionamiento competitivo respecto de las empresas de su sector, favoreciendo el efecto de captación del talento, que le permite a la organización acceder a profesionales de alta cualificación, incrementando su riqueza intelectual que directamente genera mayor capacidad competitiva que otras empresas sin dicha reputación.

A nivel interno a la propia organización, las acciones de RS tienen un efecto directo en el incremento de la reputación interna, en la creación del denominado Orgullo de Pertenencia de la persona trabajadora con la empresa, el cual posibilita la generación del contrato emocional, combinación necesaria entre conocimiento y emociones que garantizan, de una forma mucho más eficiente, los buenos resultados empresariales y personales. Asimismo, permite la retención del talento, evitando el incremento del número de fugas de las personas más valiosas y favoreciendo la autoregulación del funcionamiento de la organización.

Además del capital humano -considerado como tal los conocimiento, destrezas, habilidades y competencias derivadas de la educación, la formación y la experiencia profesional o a las personas, que aportan sus conocimientos y habilidades a las 
organizaciones-, desde la dimensión interna de la RS se considera, como afirma Salanova (2009), la garantía de un entorno de trabajo sano y seguro como uno de los aspectos básicos. Por ello es necesario promover la RS en salud y seguridad, ya que permitirá alcanzar objetivos de calidad de vida laboral más altos, al introducir acciones de promoción de la salud y mejorar la efectividad de su gestión y seguridad de los trabajadores (Betrastén y Pujol, 2003). Además, se ocupa de otros ámbitos fundamentales como la conciliación de la vida personal, familiar y laboral y la gestión de la diversidad, cuestiones de gran interés y repercusión en la situación actual de las organizaciones (Carneiro, 2004), especialmente en los momentos de crisis que España está padeciendo.

Tal y como se aprecia, el desarrollo de estrategias de RS, las cuáles se asientan sobre criterios éticos de comportamiento frente a los grupos de interés o stakeholders, tendrá dos grandes efectos en las organizaciones: sobre la competitividad, por un lado y sobre la productividad, por otro, que posibilitarán el incremento de la calidad de vida de las personas, objetivo principal en el nuevo Modelo para favorecer el desarrollo post-crisis. Porter y Kramer (2006) señalan cuatro argumentos justificadores de la RS: a) el argumento moral: aducir que las empresas tienen el deber de ser buenas ciudadanas y de "hacer lo correcto"; b) sustentabilidad: satisfacer las necesidades del presente sin comprometer la capacidad de las futuras generaciones para cubrir sus propias necesidades; c) licencia para operar: toda empresa necesita permiso tácito o explícito de los gobiernos, comunidades y muchos otros stakeholders para hacer negocios; y d) la reputación: que mejorará la imagen de una empresa, fortalecerá su marca, elevará la moral e incluso incrementará el valor de sus acciones.

En los últimos años, la idea acerca de que los esfuerzos sociales y medioambientales de las empresas serán recompensados por los consumidores y que incrementarán su visibilidad, mejorarán su imagen y las dotarán de ventajas competitivas, han conducido a que cada vez sea mayor el número de empresas que formulan y fomentan sus estrategias y acciones de negocios atendiendo a la noción de RS (Aguilera, Rupp, Willians, \& Ganapathi, 2007; Comisión de las Comunidades Europeas, 2001). Así lo reflejan los datos aportados por el Monitor IBEX RSC de 2010 que concluye que el interés de las empresas españolas por este tema es firme, pese a la crisis económica, ya que el 94\% de las compañías encuestadas cree que mantendrá e incluso aumentará la inversión en RS, así como el tamaño de los equipos dedicados a gestionarla, con propensión a aumentar las plantillas. Estos resultados ponen de manifiesto la repercusión de la RS a nivel internacional y en España y la importancia que está adquiriendo con vistas a los próximos años. Por tanto, la RS es en la actualidad una realidad, habiendo pasado de ser una moda hace apenas unos años a convertirse en un modo de entender tanto las empresas como sus relaciones con el entorno general, incluso en los tiempos actuales de crisis. 
Por tanto, según Sulbarán (1995), se ha producido una re-orientación teórica y conceptual con respecto a las respuestas de las empresas a las presiones del medio social y cultural. Este nuevo enfoque apela a la capacidad de una corporación para responder a los cambios sociales, resultando entonces evidente que la filosofía misma de la organización y la capacidad gerencial juegan un papel muy importante en la definición de cómo debe la empresa responder a las presiones y demandas de la comunidad y a la satisfacción de sus necesidades más perentorias, adquiriendo especial relevancia el estilo de liderazgo de los dirigentes de las empresas, quienes deben saber centrar sus directrices en torno a este nuevo planteamiento para llevar a cabo esta transformación de las organizaciones hacia los intereses sociales.

Pless \& Maak (2010) exponen que existe una conciencia creciente de que las relaciones con los grupos de interés son el factor más importante que determina la viabilidad de una organización (Donaldson \& Preston, 1995; Freeman, 1984; McKinsey, 2007; Wheeler \& Sillanpäa, 1997) y de que la responsabilidad social es fundamental para el éxito empresarial, tal como afirma Patrick Cescau (2007: 60), ex CEO de Unilever: "Podría decirse que actualmente hemos llegado a un punto en el que esta agenda de la sostenibilidad y de la responsabilidad corporativa no es sólo fundamental para la estrategia empresarial, sino que además se convertirá cada vez más en un factor determinante del crecimiento empresarial. Incluso se podría ir aún más lejos: en las próximas décadas lo que determinará cuáles son las empresas que tienen éxito y cuáles las que fracasan será lo bien y lo rápido que respondan a este programa”.

Estos desarrollos sitúan a los líderes ante una serie de nuevos retos interconectados (el reto de la responsabilidad, el reto de los grupos de interés y el reto de la diversidad), que comportan en todos los casos nuevas exigencias para los líderes empresariales (Pless \& Maak, 2010).

\section{ESTILOS DE LIDERAZGO FAVORECEDORES DE LA RESPONSABILIDAD SOCIAL}

Hasta el momento, en el presente artículo se ha expuesto la situación de crisis económica y social que padece España y la importancia de la RS como filosofía base del nuevo modelo que permita la recuperación de este trágico contexto. Pero sólo se ha citado la importancia del rol que deben ejercer los líderes en sus organizaciones para desarrollar este tipo de estrategias socialmente responsables, sin ahondar en dicha cuestión, acerca de la cual se profundizará en el presente apartado.

Al hablar de liderazgo en cualquier ámbito, bien sea en la organización, empresa, política, educación u otras áreas, es imposible no tocar el elemento básico de su concepción, donde líder es igual a persona que influye. Rees (1996) resalta que para 
alcanzar el éxito de un grupo debe existir un líder que, a diferencia de un simple gerente, "logra que los individuos trabajen coherentemente en proyectos definidos". A la vez refiere que esa persona a la cual todos siguen posee ciertas características que lo llevan a influir sobre el grupo. Otro concepto de este término lo ofrece Robbins (2004: 314) quien refiere que "el liderazgo se vislumbra como la capacidad de influir en un grupo para lograr el alcance de las metas". Sin embargo, no todos los líderes son jefes ni todos los jefes son líderes (Sánchez y Vecchio, 2009). Realmente no se puede ofrecer una única definición de liderazgo consensuada por todos los autores, aunque sí hay ciertas características comunes a todos ellos que resulta de especial relevancia citar: la motivación, la comunicación y la autoridad ejercida por el líder (Carreño Fernández, A. L., 2011).

Tal y como plantean Contreras, Barbosa, Juárez, Uríbe y Mejía (2009), aunque el liderazgo sea un fenómeno universal, se ha evidenciado que los valores, creencias, normas e ideales propios de una determinada cultura afectan la forma en que se ejerce el liderazgo, las metas trazadas, las estrategias utilizadas para lograrlas y la percepción que tengan los seguidores sobre el actuar del líder (Bass, 1990). Debido a ello, un estilo de liderazgo puede ser eficaz en una determinada cultura y poco eficaz en otra (Molero, 2002). Pero además, según las aportaciones de la School of Global Management and Leadership (2006), los valores específicos a nivel social y las creencias de los miembros de una cultura determinada, puede influir en los valores y creencias para el funcionamiento de las organizaciones, tales como la forma de implementar la RS.

En relación con lo anterior, Molero (2002) plantea que las relaciones entre cultura y liderazgo pueden contemplarse desde múltiples perspectivas y niveles, de manera que se pueden diferenciar, entre otras, la culturas transnacionales (las compartidas por los miembros de diferentes países), culturas nacionales (las que comparten los miembros de un mismo país), culturas subnacionales (las que son compartidas por diferentes grupos dentro de un país), culturas organizacionales (las compartidas por los miembros de una determinada organización) y culturas suborganizacionales (las compartidas por los miembros de un departamento o unidad dentro de una organización). Según esto, es de nuestra incumbencia considerar la influencia de la cultura organizacional e incluso la suborganizacional en el ejercicio del liderazgo idóneo que favorezca la implantación y el desarrollo de la RS entendida de forma general. El efecto de las características culturales sobre la gestión de las organizaciones y en particular la gestión del talento humano, ha sido estudiado asimismo por distintos autores (Bhagat Kedia, Crawford \& Kaplan, 1990; House, Wright \& Aditya, 1997; Marchese, 2001, cit. en Molero, 2002).

Estas consideraciones nos llevan necesariamente a referirnos al contexto concreto en el que nos encontremos para poder determinar el estilo de liderazgo más 
adecuado para la implantación y desarrollo de la RS. Tal y como plantean Gil, Alcover, Rico y Sánchez-Manzanares (2011), la globalización, las nuevas tecnologías de la comunicación y los cambios económicos y sociales plantean a las organizaciones nuevos roles y funciones de liderazgo. En consecuencia, la necesidad de afrontar nuevos retos exige a los líderes adoptar un papel complejo, capaz de compaginar la continuidad con el cambio y la innovación, y que a la vez esté sólidamente fundado en principios éticos y valores sociales, como los de la RS.

Salanova, Martínez y Llorens (2005) señalan que las sociedades están cambiando constantemente y con ellas, cambian también las organizaciones, lo que impacta directamente en los puestos de trabajo y por tanto, en la seguridad, la salud y el bienestar de los empleados. Si no se gestionan bien estos cambios, las organizaciones pueden enfermar, cayendo en la falta de efectividad y adaptación al entorno, como les está ocurriendo a muchas de ellas actualmente. La gestión de estos cambios continuos de la sociedad, de las organizaciones y del mercado de trabajo requiere de un cambio radical de paradigma de la Psicología, que pasa de basarse en un modelo de enfermedad (centrado en lo patológico y su reducción) a un modelo de salud, configurando lo que se llama la Psicología Positiva, que aplicada al ámbito de las organizaciones, tiene por objeto "el estudio científico del funcionamiento óptimo de las personas y de los grupos en las organizaciones, así como su gestión efectiva" (p.353). Según este enfoque, se parte de las fortalezas humanas para incrementarlas, mejorando la calidad de vida laboral y organizacional en un sentido más amplio. Si se analiza detenidamente la visión de la Psicología Organizacional Positiva, se percibe que las estrategias socialmente responsables pueden ser incluidas dentro de este paradigma, de forma que la RS desde su dimensión interna, comentada anteriormente, pretende hacer más efectiva la gestión de su personal, alcanzando su desarrollo óptimo y generando así la reputación interna y el orgullo de pertenencia. De la misma forma, la intervención sobre la dimensión externa de la RS permitirá la mejora de la reputación externa y de la competitividad de la organización, objetivo último que pretende alcanzar la Psicología Organizacional Positiva desde una visión positiva de intervención.

Este cambio de paradigma que plantea la Psicología Positiva en este contexto actual de transformación continua, requiere de nuevas formas de liderazgo que favorezcan el avance de las organizaciones hacia la excelencia empresarial, lo cual conlleva la puesta en marcha y el desarrollo de estrategias socialmente responsables. Por tanto, ¿cuál sería el liderazgo más adecuado en este contexto?

Gil y cols. (2011) proponen distintas modalidades de liderazgo que permitan responder eficazmente a las nuevas necesidades de trabajo, tales como liderazgo auténtico, liderazgo transcultural, liderazgo complejo, liderazgo en equipo, liderazgo compartido y liderazgo remoto. Pero tal y como se ha planteado anteriormente, el 
factor contexto o la influencia de la cultura donde se encuentre la organización, va a determinar en gran medida el estilo más efectivo a adoptar. Por tanto, no se puede citar un único estilo o modalidad de liderazgo que permita la implantación y el desarrollo efectivo de la RS en este contexto de cambio y de crisis en el que nos encontramos actualmente, aunque los gerentes son en gran parte responsables de la implementación de la RS (Agle et al, 1999), la cual debe estar basada en valores (Triandis, 1995; ambos presentes en School of Global Management and Leadership, 2006).

Además, como consecuencia de la que muchos consideran la peor crisis económica mundial de la historia, la confianza en los líderes de las empresas se sitúa en el nivel más bajo de los últimos años. El barómetro Edelman de confianza indica que la credibilidad de la información del máximo dirigente de una empresa se sitúa en el 17\% en Estados Unidos y no supera el 30\% en la mayoría de países, lo que supone que mucho menos de una tercera parte de los encuestados creen que lo que los líderes empresariales afirman o comunican es cierto o fiable (Edelman, 2009, cit. en Pless \& Maak, 2010).

Pless \& Maak (2010) plantean que para reconstruir la confianza en los dirigentes de las organizaciones es necesaria una actuación por parte de éstos de forma convincente y coherente y no sólo acciones de cara a la galería. Se precisan líderes que actúen de forma sostenible y no sólo empleen palabras, siendo capaces de reflexionar sobre el rol de las empresas en la sociedad y sobre la calidad de la acción socialmente responsable. Además de estas capacidades, los autores consideran que para tratar con todos los grupos de interés de las organizaciones (stakeholders), los líderes precisan tener la capacidad de gestionar y equilibrar los intereses en conflicto entre los empleados, clientes, accionistas, comunidades, gobiernos, etc., interactuando de manera efectiva. Para establecer y gestionar las relaciones con los stakeholders, hay que saber cómo identificarlos y cómo entablar un diálogo con ellos, cómo evaluar sus intereses y determinar así la legitimidad de los mismos (Mitchell, Agle \& Wood, 1997; Phillips, 2003; School of Global Management and Leadership, 2006), con un planteamiento interpersonal maduro que permita establecer y mantener relaciones de confianza (Maak, 2007; Pless, 2007), requisito indispensable para la influencia del líder sobre sus seguidores, en este caso, de los directivos sobre sus empleados.

Según Drucker (2001), aquellos que lideren los procesos de RS son quienes deben desarrollar una visión de futuro, fomenten el entusiasmo de sus seguidores y promuevan el cambio, centrando el interés de las empresas socialmente responsables hacia el factor humano como eje central. Por ello, el liderazgo efectivo no se tratará de caer bien, sino que estará definido por los resultados que se alcancen y no por los atributos de la persona en cuestión.

Incluso el propio Libro Verde de la RS pone de manifiesto que la forma en que las empresas abordan sus responsabilidades y sus relaciones con sus interlocutores, 
varía en función de diferencias sectoriales y culturales. En un primer momento, las empresas tienden a adoptar una declaración de principios, un código de conducta o un manifiesto donde señalan sus objetivos y valores fundamentales, así como sus responsabilidades hacia sus interlocutores. A continuación, estos valores han de traducirse en medidas en toda la empresa, de las estrategias a las decisiones cotidianas. Esto les obliga a añadir una dimensión social o ecológica a sus planes y presupuestos, evaluar los resultados en estos ámbitos, crear comités consultivos locales, realizar auditorías sociales o medioambientales y establecer programas de formación continua. De esta forma, el proceso de implantación de políticas de RS se va a ver influida desde sus inicios por los aspectos culturales y sectoriales en los que se encuadre la empresa, ampliando los posibles estilos de liderazgo efectivos según estas dos condiciones. Estos pasos permitirán el diseño del programa de RS, el cual significa, según Quijano Lizarazo (2007), mejorar los procesos y el bienestar de la organización para hacerla más rentable y detectar fallas que puedan convertirse en algún obstáculo a la hora de comercializar productos.

Por tanto, no es posible determinar un estilo de liderazgo efectivo para la RS en todas las culturas y sectores empresariales. Sólo se puede considerar que el liderazgo es una variable influyente en la emergencia de los valores de RS, los cuáles deben ser fundamentalmente la integridad, considerada como el estar dispuesto al intercambio de información con los seguidores, mantener sus promesas y servir a los intereses de sus seguidores y no a los de sí mismo; y liderazgo visionario, entendido como la relación entre el líder y sus seguidores basada en la generación de confianza, percepción de la inteligencia, infundir optimismo y gran respeto y admiración (Shamir et al.,1993, cit. en School of Global Management and Leadership, 2006).

El Libro Verde de la RS insiste en la importancia de la educación y la formación del personal de dirección, los empleados y otros agentes para la promoción de la RS de las organizaciones. El sistema educativo, a todos los niveles, tiene una misión fundamental en la promoción de la RS entre los ciudadanos, incluidos los que trabajan o van a trabajar en la esfera empresarial o fuera de ella. Entre los principales medios de que dispone para cumplir este papel cabe señalar las acciones que permiten a los ciudadanos comprender y apreciar los valores sociales, ecológicos o éticos y les ofrecen las herramientas adecuadas para decidir con conocimiento de causa.

Por tanto, la formación resulta necesaria tanto en las multinacionales como en las PYMES, cuyo principal obstáculo a la RS, especialmente en las de menor tamaño, es la falta de sensibilización y los recursos limitados. De esta forma, las asociaciones de pequeñas empresas, así como sus organizaciones y redes de apoyo, pueden desempeñar un papel fundamental para aumentar la sensibilización, proporcionando información y herramientas de fácil uso y difundiendo ejemplos de buenas prácticas. 


\section{CONCLUSIONES}

Como conclusión sobre el rol de liderazgo en la implantación de políticas de Responsabilidad Social, cabe hacer referencia a una serie de recomendaciones generales aplicables a todo tipo de liderazgo que Gil y cols (2011) incluyen en su trabajo. Destacan, por una parte, las que recogen Conger y Riggio (2007) en su libro 'La práctica del liderazgo: desarrollando la nueva generación de líderes'. Según estos autores, los líderes de hoy en día necesitan: 1) implicar y comprometer a los seguidores; 2) modelar el camino (visión, misión y valores); 3) actuar proactivamente; y 4) huir de atajos, porque el liderazgo efectivo es un proceso de desarrollo a largo plazo. Por otra parte, acogen a las que sugieren Kouzes y Posner (2000) acerca del liderazgo del futuro: 1) dar valor a la incertidumbre, y 2) liderar con una orientación caracterizada por la vitalidad, la improvisación, la liviandad, la autenticidad y la capacidad de aprendizaje.

Con el presente artículo se ha pretendido hacer una reflexión acerca de la situación económica-social que azota a España y de la cual es preciso emerger cuanto antes. Para ello, se ha planteado la necesidad de asentar el nuevo modelo post-crisis sobre criterios éticos de RS, cuyo desarrollo e implantación, requiere de estilos de liderazgos comprometidos con la excelencia empresarial, la globalización y el cambio.

\section{BIBLIOGRAFÍA}

Aguilera, R.V., Rupp, D., Willians, C. \& Ganapathi, J. (2007). "Putting the back in CSR: A multi-level theory of social change in organizations". Academy of Management Review, 32 (3), pp. 836-863.

Alabort, E., Buendia, L., Fuster, G., Obispo, M., y Rodríguez, Ll. (2008). "Crisis Económica y Resistencia Obrera: la Crisis Mundial y sus Efectos en España”. Segunda parte del resumen del número 1. Cuadernos del Instituto de Ciencias Económicas y de la Autogestión (ICEA). Gaceta sindical y económica del Periódico, n³51, diciembre.

Betrastén, M. y Pujol, L. (2003). Responsabilidad Social de las empresas: tipos de responsabilidades y plan de actuación. Madrid: Ministerio de Trabajo y Asuntos Sociales.

Carneiro, M. (2004). La Responsabilidad Social Corporativa Interna. La "nueva frontera" de los Recursos Humanos. Madrid: ESIC.

Carreño Fernández, A. L. (2011). El liderazgo en la sociedad actual: Valores. II Jornadas de Liderazgo. Cátedra General Castaño. Ejército Terrestre. Celebradas en Sevilla, 9-10 noviembre 2011.

Comisión de las Comunidades Europeas (2001). Libro Verde sobre la Responsabilidad Social de las empresas. Bruselas: Comisión Europea. 
Contreras, F., Barbosa, D., Juárez, A. F., Uríbe, A. F., y Mejía, C. (2009). "Estilos de Liderazgo, Clima Organizacional y Riesgos Psicosociales en entidades del sector de la salud. Un estudio comparativo". Acta Colombiana de Psicología, 12(2), pp. 13-26.

De Castro Sanz, M. (2005). "La Responsabilidad Social de las Empresas, o un Nuevo Concepto de Empresa”. CIRIEC. Revista de Economía Pública, Social y Cooperativa, 53, pp. 29-51.

De la Corte, J. (2011). "Competitividad y Responsabilidad Social en las Empresas: Especial Significación en las PYMES”. En Gómez Muñoz, J. M., Valdés Alonso, A., Gala Durán, C., De la Corte, J., Morales Ortega, J. M., Pérez Yañez, R. M., Castellano Burguillo, E., Sánchez Huete, M. A., Fernández Amor, J. A., Fernández Villarino, R., y Venero Valenzuela, J. P. (autores). Estudios en Materia de Responsabilidad Social Empresarial. Editorial: CINCA. pp. 138-165. Manuscrito presentado para publicación.

Directrices Generales para las Políticas Económicas de los Estados miembros y de la Unión (2010). Recomendación del Consejo. 2010/410/UE. Diario oficial de la Unión Europea.

Drucker, P. (2001). The essential Drucker. New York: Harper Collins Publishers.

García-Trevijano (2011). "Efectos de la Crisis Económica". Diario Español República Constitucional. Año IV no 498. Recuperado el día 15 de septiembre de 2011 de: http://diariorc.com/efectos-de-la-crisis.html

Garst, H., Frese, M., \& Molenaar, P. (2000). "The temporal factor of change in stressorstrain relationships: a growth curve model on a longitudinal study in East Germany". Journal of Applied Psychology, 85(3), pp. 417 - 38.

Gil, F., Alcover, C. M., Rico, R., y Sánchez-Manzanares, M. (2011). "Nuevas formas de liderazgo en equipos de trabajo”. Papeles del Psicólogo, 32 (1), pp. 38-47.

González, T. (2003). "La Responsabilidad Social de la Empresa: Un buen negocio". Recuperado el 20 de junio de 2011 de: http://www.monografias.com/ trabajos13/ bune/ bune2.html

Hans de Witte (2011). Job Insecurity: An overview of past and future research (and some exercises). Acto de clausura del Master en Gestión y Desarrollo de los Recursos Humanos, Septiembre, Universidad de Sevilla. No publicado.

Instituto Nacional de Estadística (2011). Encuesta Población Activa en España. Tercer Trimestre.

Molero, F. (2002). "Cultura y Liderazgo. Una relación Multifacética”. Boletín de Psicologia, 76, pp. 53-75. 
Monitor IBEX RSC (2010). Los directores de RSC de las empresas del IBEX 35 opinan (2010). España: KPMG.

Pless, N. M. \& Maak, T. (2010). "Desarrollando Líderes Globales Responsables". Universia Business Review. Tercer Trimestre, pp. 58-71.

Porter, M. E. y Kramer, M. R. (2006). "Estrategia y Sociedad". Harvard Business Review, 84(12), pp. 78-92.

Quijano Lizarazo, A. (2007). "Guía práctica de desarrollo de un programa de Responsabilidad Social”. Consultado en http://www.larepublica.com.co/RSE/articulos.html.

Sánchez, M. y Vecchio, F. (2009). Liderazgo para la comunicación eficaz de la responsabilidad social empresarial en el Rotary Club. GT Gestión de la Comunicación en la Organización: Identidad e Imagen Corporativa.

Salanova, M. (2009). "Organizaciones Saludables: Una aproximación desde la Psicología Positiva”. En Vázquez, C., y Hervás, G. (Eds.), Psicología Positiva: Bases científicas del bienestar y la resiliencia. Madrid: Alianza Editorial, pp. 2-32.

Salanova, M., Martínez, I. M. y Llorens, S. (2005). "Psicología Organizacional Positiva". En Palací Descals, F. J. (Ed.) Psicología de la Organización. Pearson Prentice Hall, pp. 349-376.

School of Global Management and Leadership (2006). "Cultural and leadership predictors of corporate social responsibility values of top management: a GLOBE study of 15 countries". Journal of International Business Studie 37, pp. 823-837.

Sulbarán, J. P. (1995). "El concepto de responsabilidad social en la empresa". Revista Economía, 10, pp. 225-248.

Vargas Niello, (2006). Responsabilidad Social Empresarial (RSE) desde la Perspectiva de los Consumidores. Comisión Económica para América Latina y el Caribe (CEPAL): Naciones Unidas.

V Encuesta Europea de Condiciones de Trabajo (2010). European Foundation for the Improvement of Living and Working Conditions. Traducido por Agustín González García. España: Instituto Sindical de Trabajo, Ambiente y Salud (ISTAS). 
